\title{
HIF1A activates the transcription of IncRNA RAET1K to modulate hypoxia-induced glycolysis in hepatocellular carcinoma cells via miR-100-5p
}

\author{
Yufan Zhou', Yun Huang ${ }^{1}$, Kuan Hu' ${ }^{1}$ Zeyu Zhang ${ }^{1}$, Jiajin Yang ${ }^{1}$ and Zhiming Wang ${ }^{1}$
}

\begin{abstract}
Hepatocellular carcinoma (HCC) remains the primary cause of cancer-related death. Metabolic change is the major characteristic of cancer. The present study attempted to investigate the regulatory mechanisms of HCC energy metabolism from the perspective of noncoding RNA regulation of HIF1A and LDHA. The expression of miR-100-5p expression was significantly suppressed in HCC tissue samples and HCC cell lines under $1 \% \mathrm{O}_{2}$-induced hypoxia. miR100-5p overexpression significantly suppressed hypoxia-induced increases in lactate concentration and glucose uptake. Exposure to $1 \% \mathrm{O}_{2}$ induced HIF1A protein and reduced miR-100-5p expression, while HIF1A silencing dramatically rescued miR-100-5p expression upon $1 \% \mathrm{O}_{2}$ exposure. In addition, $1 \% \mathrm{O}_{2}$-induced increases in lactate concentration and glucose uptake were also suppressed by HIF1A silencing. Next, by analyzing available data in TCGA, we found that InCRNA RAET1K was correlated with HIF1A and miR-100-5p.LncRNA RAET1K could downregulate the expression of miR-100-5p by acting as a sponge, while HIF1A bound the IncRNA RAET1K promoter region to activate its transcription. LncRNA RAET1K silencing significantly suppressed HCC cell proliferation and invasion and also suppressed hypoxia-induced increases in lactate concentration and glucose uptake, while miR-100-5p inhibition reversed the effects of IncRNA RAET1K silencing on hypoxia-induced glycolysis in HCC cells. Finally, the expression of HIF1A, IncRNA RAET1K, and LDHA was upregulated in HCC tissue specimens; the expression of miR-100-5p was negatively related to HIF1A, InCRNA RAET1K, and LDHA; and HIF1A, InCRNA RAET1K, and LDHA were positively correlated with each other. In conclusion, the HIF1A/IncRNA RAET1K/miR-100-5p axis modulates hypoxia-induced glycolysis in HCC cells and might affect HCC progression.
\end{abstract}

\section{Introduction}

Globally, hepatocellular carcinoma (HCC) is not only the most common type of digestive system tumor but also the primary cause of death from cancer $^{1}$. The 5 -year survival rate of patients with HCC remains low due to late diagnosis, intrahepatic metastasis, and recurrence after operation $^{2}$. It is urgent to fully understand the molecular mechanisms of HCC development.

One of the characteristic features of tumor cells is metabolic change ${ }^{3}$. Instead of depending on mitochondrial

Correspondence: Zhiming Wang (13808462382@163.com)

'Department of Hepatobiliary Surgery, Xiangya Hospital, Central South University, Changsha 410008, China

Edited by G. Calin oxidative phosphorylation, cancer cells are more likely to produce energy via glycolysis. This phenomenon, known as the Warburg effect, usually leads to increased in glucose uptake, ATP accumulation, and production of lactic acid in cancer cells ${ }^{4}$. Normally differentiated hepatocytes cannot produce energy through aerobic glycolysis in the non-anaerobic environment. However, in HCC, the metabolic pathways are extensively reprogrammed ${ }^{5,6}$. Tumor cells prefer hypoxic environments and use glycolysis to produce $\mathrm{ATP}^{4,7}$. Therefore, focusing on HCC metabolism, especially under low oxygen conditions, may contribute to the treatment of HCC. A a subunit of the heterodimeric transcription factor hypoxia-inducible factor-1 (HIF1), HIF1A is expressed in several human 
malignancies ${ }^{8,9}$. More importantly, lactate dehydrogenase A (LDHA) is not only a member of the tetrameric enzyme lactate dehydrogenase (LDH) family ${ }^{10}$ and an essential component of the last step of the glycolytic pathway but also exerts critical effects on tumor maintenance and could be regulated by HIF $1^{11-13}$. Therefore, we attempted to investigate the regulatory mechanisms of HCC energy metabolism from the perspective of HIF1A and LDHA regulation.

Recently, emerging evidence has indicated the importance of noncoding RNAs, such as long noncoding RNAs (more than 200 nucleotides in length) ${ }^{14}$ and micro RNAs (miRNAs), which are evolutionally conserved, small noncoding RNAs that could inhibit translation and/or induce mRNA degradation to modulate gene expression at the posttranscriptional level ${ }^{15}$. Noncoding RNAs participate in the modulation of various biological processes of cancer, including glucose metabolism ${ }^{16}$, via a wellestablished mechanism by which lncRNAs regulate miRNAs by serving as competing endogenous RNAs (ceRNAs) ${ }^{17,18}$. Among deregulated miRNAs in HCC, miR100-5p was shown to be downregulated in HCCs of humans and rats. The downregulation of miR-100-5p is an initiating event in HCC, and miR-100-5p downregulation is not only related to all phases of $\mathrm{HCC}^{19}$, but is also associated with tumor development and poor prognosis of HCC patients ${ }^{20}$. More importantly, miR-100-5p expression has been reported to be downregulated by long-term hypoxia in clear cell renal cell carcinoma ${ }^{21}$. In non-muscle invasive bladder cancer, hypoxia suppressed miR-100-5p expression to induce FGFR3 expression, therefore promoting cell growth ${ }^{22}$. Even so, the function and mechanism by which miR-100-5p exerts its biological effects on glycolysis in HCC in a hypoxic microenvironment remain unclear.

The study first examined miR-100-5p expression in HCC tissue samples and cell lines under hypoxia $\left(1 \% \mathrm{O}_{2}\right)$, as well as its effect on lactate concentration and glucose uptake under hypoxia. We also detected how HIF1A affected the expression of miR-100-5p under hypoxia. Next, we analyzed the available data in the TCGA database to identify lncRNAs that might be related to both HIF1A and miR-100-5p, and lncRNA RAET1K (isoform 202) was selected. The predicted binding between HIF1A and IncRNA RAET1K, and between IncRNA RAET1K and miR-100-5p was validated. Furthermore, the effect of IncRNA RAET1K on the proliferation and invasion of HCC cells, and the dynamic effects of lncRNA RAET1K and miR-100-5p on glycolysis were examined under hypoxia. Finally, the expression of HIF1A, lncRNA RAET1K, and LDHA in tissue samples was determined, and the correlations of HIF1A, IncRNA RAET1K, LDHA, and miR-100-5p were analyzed. In summary, this study demonstrated the role and the mechanism of the HIF1A/ IncRNA RAET1K/miR-100-5p axis in the regulation of HCC glycolysis under hypoxia.

\section{Materials and methods \\ Clinical tissue specimens}

A total of 66 paired HCC and adjacent normal tissues were collected from patients (with clear pathological diagnoses) who underwent primary surgical resection at the Xiangya Hospital of Central South University (Changsha, China) with approval by the Research Ethics Committee of the Xiangya Hospital of Central South University (Changsha, China), and written informed consent was obtained from all patients.

\section{Cell lines, cell culture, and cell transfection}

The L02 cell line was obtained from China Center for Type Culture Collection (CCTCC; Wuhan, China) and cultured in the MEM-EBSS: Minimum Essential Medium (MEM with Earle's Balanced Salts) supplemented with 10\% FBS (Invitrogen, Waltham, MA, USA). The HCCLM3 (Cat. CL-0278) cell lines were obtained from ProCell (Wuhan, China) and cultured in RPMI-1640 medium supplemented with $10 \%$ FBS (Invitrogen). HepG2 cell line was obtained from ATCC (ATCC ${ }^{\circledR}$ HB$8065^{\mathrm{TM}}$, Manassas, VA, USA) and cultured in MEM (ATCC) supplemented with 10\% FBS (Invitrogen). The huh7 cell line was obtained from CCTCC (Cat. GDC0134) and cultured in RPMI-1640 medium supplemented with 10\% FBS (Invitrogen). The Hep3B cell line was obtained from ATCC (ATCC ${ }^{\oplus} \mathrm{HB}-8064^{\mathrm{TM}}$ ) and cultured in MEM (ATCC) supplemented with 10\% FBS (Invitrogen). Cells were cultured in $5 \% \mathrm{CO}_{2}$ at $37^{\circ} \mathrm{C}$. For hypoxia treatment, cells were exposed to $1 \% \mathrm{O}_{2}$ for $12 \mathrm{~h}$ and then switched to normoxia for an additional $24 \mathrm{~h}$ using a tri-gas incubator (SANYO, Japan). Then, cells were harvested for further experiments.

The miR-100-5p mimics, miR-100-5p inhibitor, as well as their negative controls (mimics-NC and inhibitor-NC), were purchased from Genepharma (Shanghai, China). SiHIF1A\#1 (HIF1A 1418-1140) (sense 5'- GCUAUUCAC CAAAGUUGAATT -3'; antisense 5'- UUCAACUUUG GUGAAUAGCTT -3'), si-HIF1A\#2 (HIF1A 2271-2293) (sense $5^{\prime}$ - CCAUAUAGAGAUACUCAAATT - $3^{\prime}$; antisense $5^{\prime}$ - UUUGAGUAUCUCUAUAUGGTT $-3^{\prime}$ ) and a negative control (si-NC; sense: $5^{\prime}$-UUC UCC GAA CGU GUC ACG UTT - $3^{\prime}$; antisense: $5^{\prime}$-ACG UGA CAC GUU CGG AGA ATT $-3^{\prime}$ ) were also purchased from Genepharma (A01003, Shanghai, China). The IncRNA RAET1K smart silencer (a pool containing three siRNAs and three antisense oligonucleotides) and its negative control, lncRNA smart silencer NC, were purchased from RiboBio (siBDM1999A, Guangzhou, China). For RAET1K 
overexpression, the whole length of RAET1K fragment was cloned into the pcDNA3.1 expression vector by PCR. The primers and siRNA sequences are listed in Table S1. All transfections were performed by using Lipofectamine 3000 reagent (Invitrogen, CA, USA) according to the manufacturer's instructions.

\section{RNA fluorescence in situ hybridization}

A Specific FAM-labeled lncRNA RAET1K probe and FITC-labeled miR-100-5p probe were obtained from RiboBio (Guangzhou, China). Cells were fixed in $4 \%$ formaldehyde for $30 \mathrm{~min}$ and then washed with PBS. After permeabilization, cells were prehybridized with hybridization solution and then incubated with both the lncRNA and miRNA probes in hybridization buffer. Cell nuclei were stained with DAPI for $5 \mathrm{~min}$ at room temperature. Fluorescence images were obtained by fluorescence microscopy (Olympus, Japan). The probe sequences were listed in Table S1.

\section{Xenograft nude mice}

AntagomiR-100-5p, antagomiR-NC, shRNA negative control vector (lv-sh-NC), and sh-lncRNA RAET1K (shRAET1K) vector were obtained from GENECHEM (Shanghai, China). AntagomiR-100-5p, antagomiR-NC sh- RAET1K or sh-NC was transfected into HCCLM3 cells with Lipofectamine 2000. Forty-eight hours later, the transfected cells were harvested for subcutaneous injection. Six-week-old female nude mice (BALB/c-nu/nu) weighing $16-20 \mathrm{~g}$ were obtained from the Xiangya Experimental Animal Center (Changsha, China). All experimental procedures were approved by the Ethic Committee of Xiangya Hospital of Central South University. Mice were randomly divided into 4 groups: Control (no transfection) group, sh-NC + antagomiR-NC group, sh-RAET1K + antagomir-NC group and shRAET1K + antagomiR-100-5p group. A total of $1 \times 10^{6}$ transfected HCCLM3 cells were suspended in $100 \mu \mathrm{l}$ of PBS and subcutaneously injected into the left anterior armpits of the nude mice. Twenty-eight days later, the nude mice were sacrificed. The tumor weight and volume were measured. The expression of LDHA was measured by immunohistochemistry (IHC).

\section{RNA isolation and qRT-PCR}

RNA isolation and qRT-PCR were performed as described previously ${ }^{23}$ using a TaqMan MicroRNA Reverse Transcription Kit (4366596, ThermoFisher, CA, USA) or a PrimeScript reverse transcriptase reagent kit (Takara, Osaka, Japan). U6 or Tubulin was used as an internal control. The expression levels were normalized to $\mathrm{U} 6$ or Tubulin to produce a $2^{-\Delta \Delta \mathrm{Ct}}$ value for the relative expression level. The primers are listed in Table S1.

\section{Immunoblotting analyses}

Immunoblotting analyses were performed to examine protein levels. We separated proteins by SDS-PAGE and transferred proteins to PVDF membranes. The experimental details was performed similarly to previously reported $^{24,25}$. The following primary antibodies were used: anti-LDHA (ab101562, Abcam, Cambridge, MA, USA), anti-HIF1A (ab51608, Abcam), anti-GAPDH (ab181602, Abcam) and anti- $\beta$-actin (ab8226, Abcam).

\section{Immunohistochemical staining}

The tumor tissues and xenograft tumor tissues were paraffin-embedded and cut into $3-\mu \mathrm{m}$ sections and mounted on glass slides for staining with immunoperoxidase, and IHC was performed as previously described $^{24,25}$. The following antibodies were used: anti-LDHA (ab101562, Abcam) and anti-HIF1A (ab51608, Abcam). The pictures were visual under a microscope (Olympus, Japan).

\section{MTT assay}

Cell viability was determined following methods described previously using a cell proliferation kit (C0009, Beyotime, China $)^{26}$. The OD value was measured at $490 \mathrm{~nm}$. The viability of the nontreated cells (control) was defined as $100 \%$, and the viability of cells was calculated based on that of the control group.

\section{DNA synthesis determination by a 5-bromo-2- \\ deoxyUridine incorporation assay}

DNA synthesis in proliferating cells was determined by measuring 5-bromo-2-deoxyUridine (BrdU) incorporation following methods described previously ${ }^{27}$ with a peroxidase-coupled anti-BrdU-antibody (MAB3510P, Sigma-Aldrich). The absorbance values were measured at $450 \mathrm{~nm}$. The background BrdU OD value was determined in cells not exposed to BrdU but stained with the BrdU antibody.

\section{Luciferase reporter assay}

The fragment of IncRNA RAET1K or LDHA-3'UTR was amplified and cloned downstream of Renilla in the psiCHECK2 vector (C8021, Promega, Madison, WI, USA), and the construct was named wt-RAET1K or wtLDHA. To generate the IncRNA RAET1K or LDHA mutant reporter, we mutated the seed region of lncRNA RAET1K or LDHA-3'UTR to remove the complementarity to miR-100-5p and named the construct mut-RAET1K or mut-LDHA. HEK293 cells (ATCC, USA) were seeded into a 24-well plate and cotransfected with the indicated vectors, and miR-100-5p mimics or miR-100-5p inhibitor. Luciferase assays were performed $48 \mathrm{~h}$ after transfection using the Dual-Luciferase Reporter 
Assay System (Promega). Renilla luciferase activity was normalized to firefly luciferase activity for each transfected well.

To confirm the binding between HIF1A and IncRNA RAET1K, we cotransfected the cells with wt-RAET1K or mut-RAET1K, a pGL3 luciferase reporter construct harboring the HIF1A response element (HIF1A-RE) target sequence and si-NC or si-HIF1A. After $24 \mathrm{~h}$, the activities of firefly luciferase and Renilla luciferase were measured in the cell lysates using the Dual-Luciferase Assay System (Promega, WI, USA).

\section{Chromatin immunoprecipitation}

Cells were transfected or treated, cross-linked by $1 \%$ formaldehyde, and DNA was sheared to an average fragment of $400 \mathrm{bp}$, and immunoprecipitated using antibody against HIF1A (anti-HIF1A, ab1, Abcam). The chromatin immunoprecipitation (ChIP)-PCR primers were designed to amplify the promoter regions containing putative HIF1A binding sites within IncRNA RAET1K. A positive control antibody (against RNA polymerase II) and a negative control nonimmune IgG were used to demonstrate the efficacy of the kit reagents (P-2025-48, Epigentek Group Inc., NY, USA). The immunoprecipitated DNA was subsequently cleaned, and eluted. Specific PCR primers were used to determine the level of VEGF and RAET1K promoter fragments in the immunoprecipitated DNA. The fold-enrichment (FE) was calculated as the ratio of the amplification efficiency of the ChIP sample to that of the nonimmune IgG. The amplification efficiency of RNA Polymerase II was used as a positive control. FE\% $=2($ IgG CT-Sample CT $) \times 100 \%$. The primer sequences were listed in Table S1.

\section{Measurement of glucose and lactate}

Glucose levels were determined using a commercial glucose assay kit, Glucose Colorimetric assay kit II (K686100, Bio Vision, Milpitas, CA, USA). Glucose uptake was calculated by deducting the detected glucose concentration in the medium from the original glucose concentration. Lactate levels were determined using a lactate colorimetric assay kit (K627-100, Biovision, Milpitas, CA, USA) in accordance with the manufacturer's instructions. Considering that the cell number in every sample may be different, all the concentrations of glucose or lactate production were finally normalized to the total cell protein concentration. The protein concentration was measured by the BCA protein assay (Cat. 23225, Thermo Fisher Scientific, Waltham, MA, USA).

\section{Statistical analysis}

All data from three independent experiments were expressed as mean $\pm \mathrm{SD}$ and processed using SPSS17.0 statistical software. The differences between two groups were estimated by Student's $t$-test; the differences among more than two groups were estimated by one-way ANOVA. A $P$ value of $<0.05$ was considered to be statistically significant.

\section{Results \\ Hypoxic environment promotes glycolysis by inhibiting miR-100-5p and promoting LDHA expression}

To confirm that miR-100-5p participates in the regulation of glycolysis, the study first examined miR-100-5p expression in tissue samples or in response to hypoxic conditions. The expression of miR-100-5p in HCC tissue samples was significantly downregulated, compared to that in noncancerous tissues $(n=66$, Fig. 1a). The fold change of miR-100-5p was represented as $\log _{2}$ (tumor/ normal) in Fig. 1b. Data from the TCGA online database showed that a higher miR-100-5p expression was correlated with higher survival probability (Fig. 1c).

Next, the expression of miR-100-5p was determined in four HCC cell lines, HCCLM3, Huh7, Hep3B, and HepG2, and the normal cell line L02. As shown in Fig. 1d, miR100-5p expression was significantly downregulated in all four HCC cell lines compared to L02 cells. Then, HCCLM3, Huh7, Hep3B, and HepG2 cells were exposed to $1 \% \mathrm{O}_{2}$ to mimic hypoxic conditions, and miR-100-5p expression was examined. As shown in Fig. 1e, miR-100$5 \mathrm{p}$ expression was significantly suppressed by hypoxia in all four HCC cell lines and was the most suppressed in HCCLM3 and HepG2 cells; thus, HCCLM3 and HepG2 cells were used in further analyses. We modified miR-100$5 p$ expression by transfecting HCCLM3 and HepG2 cells with miR-100-5p mimics/miR-100-5p inhibitor, and performed real-time PCR to verify the transfection efficiency (Fig. S1A). miR-100-5p-overexpressing HCCLM3 and HepG2 cells were then examined for lactate concentration and glucose uptake under normoxia or hypoxic condition. The lactate concentration and glucose uptake were significantly induced by hypoxia but inhibited by miR-100-5p overexpression (Fig. 1f, g), suggesting that miR-100-5p overexpression might block glycolysis in cancer cells in a hypoxic microenvironment. Moreover, BrdU assays performed under the same conditions indicated that tumor cell viability was promoted by hypoxia but significantly suppressed by miR-100-5p overexpression (Fig. 1h). These data suggest that miR-100-5p might modulate HCC cell phenotypes by affecting glycolysis under hypoxia.

More importantly, there was a negative association between miR-100-5p expression and LDHA expression (Fig. 1i). As a member of the LDH family of tetrameric enzymes ${ }^{10}$, LDHA is not only a vital component of the last step of the glycolytic pathway but also exerts significant effects on tumor maintenance, according to the TCGA online database. Additionally, as shown by the TCGA analysis, higher LDHA expression was correlated with 


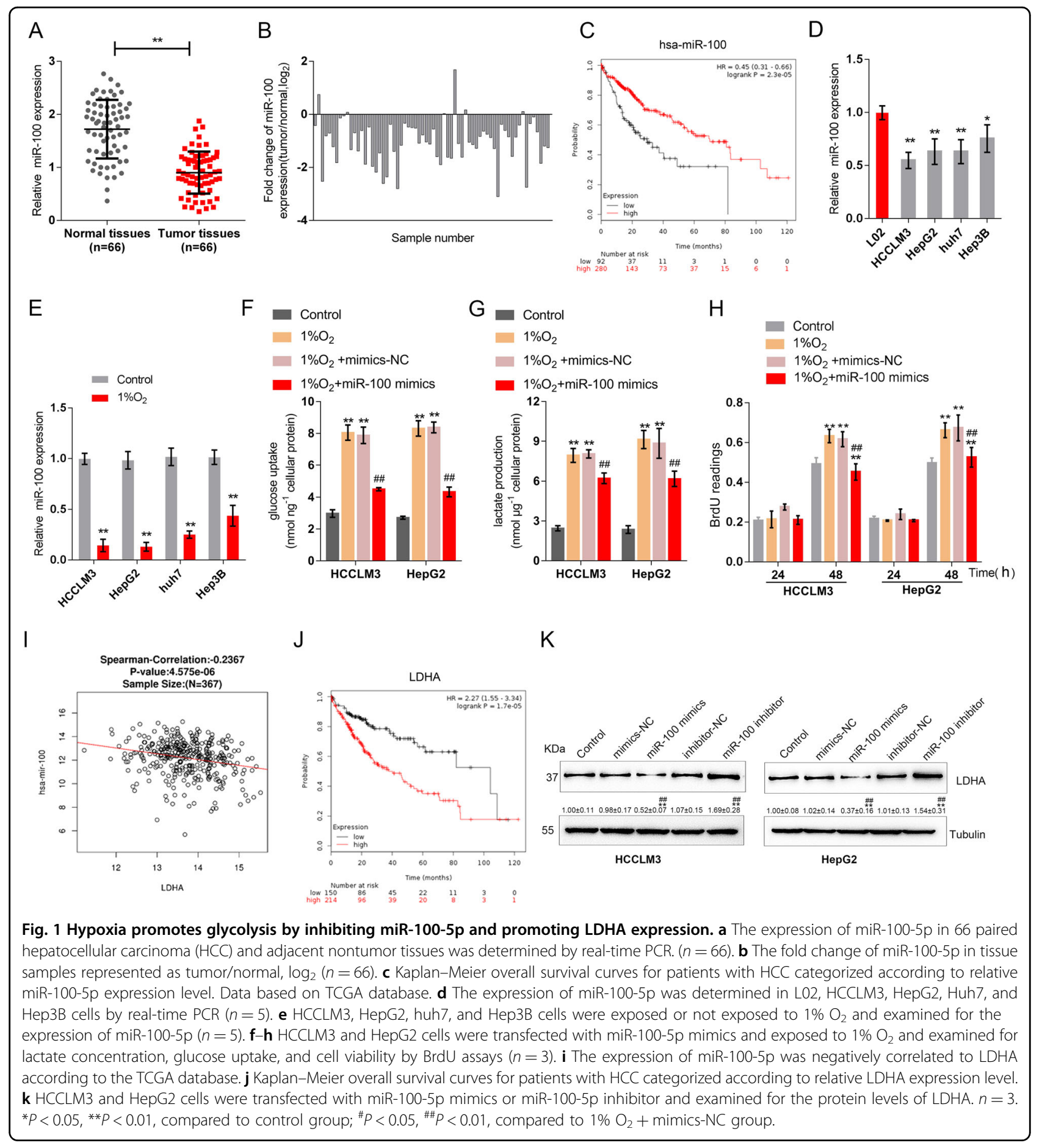

lower survival probability (Fig. 1j). Additionally, in HCCLM3 and HepG2 cells, miR-100-5p overexpression downregulated, while miR-100-5p inhibition upregulated LDHA protein levels (Fig. 1k). To further confirm the negative correlation between miR-100-5p and LDHA, a luciferase reporter assay was performed by constructing two different LDHA 3'UTR luciferase reporter vectors, wild-type (wt-LDHA $3^{\prime}$ UTR) and mutant-type (mut-
LDHA 3'UTR) (Fig. S1B). These vectors were cotransfected into 293T cells with miR-100-5p mimics or miR$100-5 p$ inhibitor, and the luciferase activity was determined. As shown in Fig. S1C, the luciferase activity of wtLDHA 3'UTR was significantly suppressed by miR-100-5p overexpression but increased by miR-100-5p inhibition. In addition, after mutating the predicted miR-100-5p binding site, the changes in luciferase activity were 


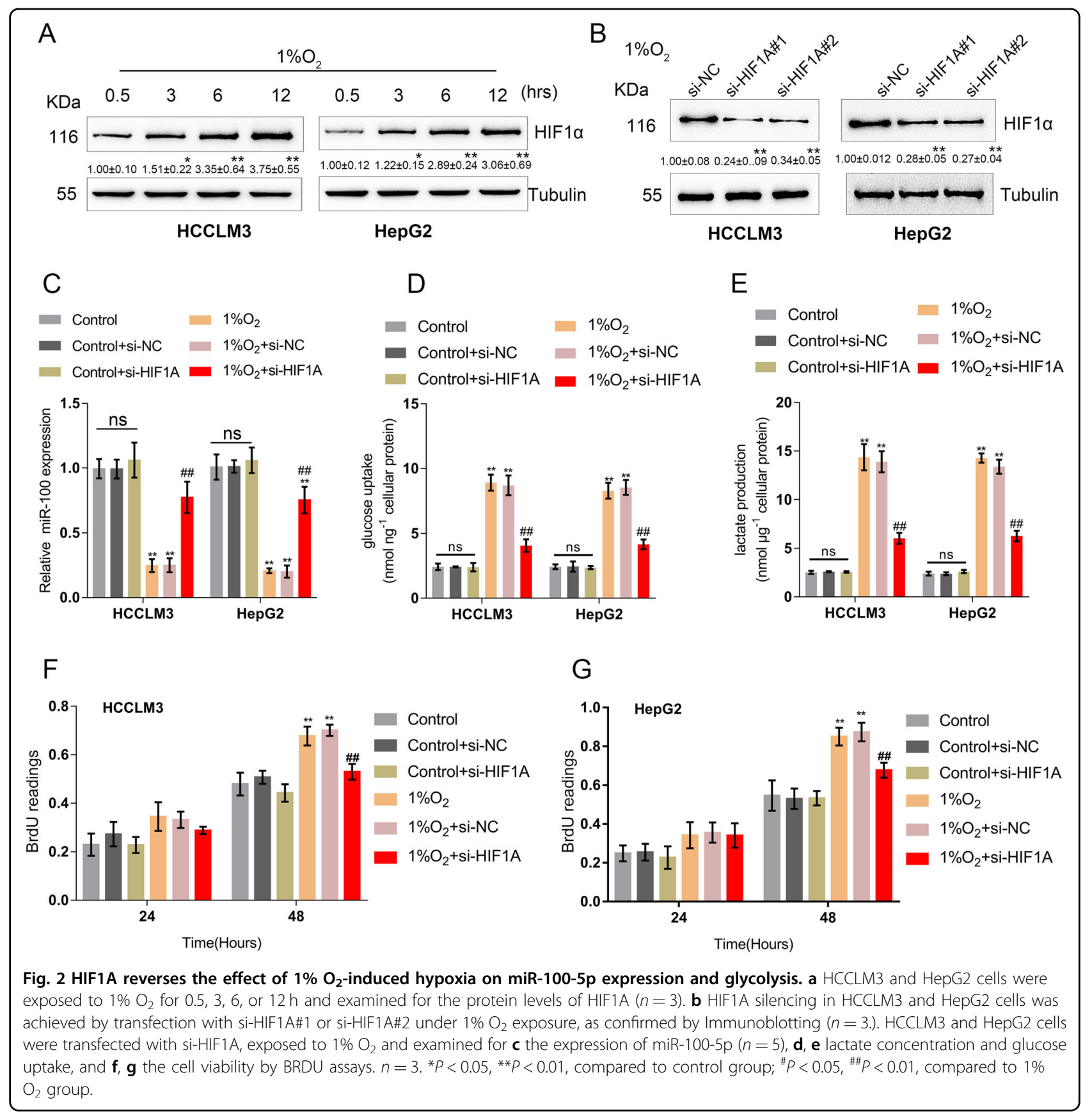

abolished (Fig. S1C). Moreover, as shown in Fig. S2, miR100-5p overexpression significantly decreased while miR100-5p inhibition increased lactate concentration and glucose uptake in HCC cells. In summary, miR-100-5p exerts its biological effect on glycolysis in HCC cells under hypoxic conditions $\left(1 \% \mathrm{O}_{2}\right)$.

HIF1A reverses the effect of $1 \% \mathrm{O}_{2}$-induced hypoxia on miR-100-5p expression and glycolysis

HIF triggers LDHA upregulation, while LDHA and HIF1A form a positive feedback loop leading to an increase in lactic acid concentration ${ }^{28}$. Next, we investigated whether HIF1A could affect the inhibitory effect of hypoxia on miR-100-5p expression. In HCCLM3 and HepG2 cells, the protein levels of HIF1A were significantly induced by $1 \% \mathrm{O}_{2}$ exposure in a time-dependent manner (Fig. 2a). Under $1 \% \mathrm{O}_{2}$-induced hypoxic conditions, HIF1A silencing was achieved in HCCLM3 and HepG2 cells by transfection with si-HIF1A, as confirmed by immunoblotting (Fig. 2b). HIF1A overexpression was generated by the transfection of the HIF1Aoverexpressing vector, as verifed by immunoblotting 
(Fig. S3). As shown in Fig. 2c, $1 \% \mathrm{O}_{2}$-induced suppression of miR-100-5p expression was significantly reversed by HIF1A silencing in both HCCLM3 and HepG2 cell lines. In addition, $1 \% \mathrm{O}_{2}$-induced increases in lactate concentration and glucose uptake were reduced considerably by HIF1A silencing (Fig. 2d, e), indicating that HIF1A could affect the expression of miR-100-5p, therefore modulating the glycolysis in $\mathrm{HCC}$ cells under $1 \% \mathrm{O}_{2^{-}}$ induced hypoxia. Similarly, BrdU assays suggested that $1 \%$ $\mathrm{O}_{2}$-induced HCC cell proliferation could be significantly suppressed by HIF1A silencing (Fig. 2f, g).

\section{The HIF1A/IncRNA RAET1K axis regulates miR-100-5p expression}

In addition to those of miRNAs, the roles of lncRNAs in glycolysis have been reported ${ }^{29-31}$. LncRNAs could suppress the expression of miRNAs and exert their effect on miRNA functions by acting as miRNA sponges through direct targeting ${ }^{32}$. Next, we analyzed data in TCGA to identify lncRNAs that are negatively correlated with miR100-5p and positively correlated with HIF1A, and lncRNA RAET1K was selected. Moreover, a higher expression of lncRNA RAET1K was associated with lower survival probability (Fig. S4). Consistent with these online data, miR-100-5p could negatively modulate lncRNA RAET1K expression in HCCLM3 and HepG2 cell lines (Fig. 3a). To investigate the effects of IncRNA RAET1K, we transfected cells with si-RAET1K to silence lncRNA RAET1K, and performed real-time PCR to verify the transfection efficiency (Fig. 3b).The results showed that LncRNA RAET1K silencing could negatively modulate miR-100-5p expression in HCCLM3 and HepG2 cell lines (Fig. 3c), indicating that IncRNA RAET1K could indeed act as a sponge of miR-100-5p. In addition, RAET1K overexpression reduced the miR-100-5p level in HCCLM3 and HepG2 cell lines further confirmed the sponge effect of RAET1K on miR100-5p (Fig. S5A, B). More importantly, HIF1A overexpression under normoxia significantly increased lncRNA RAET1K expression (Fig. 3d), while the $1 \% \mathrm{O}_{2^{-}}$ induced increase in IncRNA RAET1K expression was considerably inhibited by HIF1A silencing (Fig. 3d), indicating that HIF1A might affect IncRNA RAET1K expression, subsequently modulating miR-100-5p expression under hypoxia. As expected, HIF1A overexpression significantly inhibited, while lncRNA RAET1K silencing promoted the expression of miR-100-5p; lncRNA RAET1K significantly reversed the effect of HIF1A overexpression on miR-100-5p expression (Fig. 3e).

To investigate the molecular mechanism, we performed luciferase reporter assays to examine the putative binding between IncRNA RAET1K and miR-100-5p predicted by an online tool (Fig. 3f). We constructed two lncRNA RAET1K luciferase reporter vectors, wild-type and mutant-type RAET1K. In the putative miR-100-5p binding site of mutant-type RAET1K reporter vector, 5 bases were mutated (Fig. 3f). After cotransfection, the luciferase activity of the wt-RAET1K vector was remarkably downregulated by miR-100-5p overexpression, whereas it was upregulated by miR-100-5p inhibition, and mutating the putative miR-100-5p binding site eliminated the changes in luciferase activity (Fig. 3g). Moreover, the fluorescence in situ hybridization (FISH) results further confirmed the colocation of IncRNA RAET1K and miR100-5p in the cytoplasm (Fig. 3h). The lncRNA RAET1K promoter region also contained a HIF1A response element (HIF1A RE); thus, the study performed luciferase reporter assays to examine the binding between HIF1A and lncRNA RAET1K by constructing lncRNA RAET1K reporter vectors containing wild-type or mutated HIF1A binding sites (Fig. 3i). We cotransfected these vectors into HEK293 cells along with si-HIF1A under hypoxia. The luciferase activity of wt-RAET1K was significantly suppressed by HIF1A silencing, while mutating the putative HIF1A binding site was eliminated the changes in luciferase activity (Fig. 3j). To provide further evidence on the binding, we used a HIF1A antibody to conduct the ChIP assay under hypoxia. As shown in Fig. 3k, the level of HIF1A antibody binding to HIF1A RE within the lncRNA RAET1K promoter was significantly increased compared to that of IgG and was similar to that of VEGF, a widelyrecognized HIF1A target gene ${ }^{33}$. These data indicate that HIF1A directly targets lncRNA RAET1K, while lncRNA RAET1K directly targets miR-100-5p to form a regulatory axis that could modulate glycolysis in HCC cells under hypoxia.

\section{Effects of IncRNA RAET1K on the proliferation and invasion of HCC cells}

Next, we evaluated how IncRNA RAET1K silencing affected the ability of HCC cells to proliferate and invade. According to Fig. 4a-c, lncRNA RAET1K silencing significantly inhibited the growth and DNA synthesis capacity of HCC cell lines. Similarly, lncRNA RAET1K silencing also remarkably inhibited HCC cell invasion (Fig. 4d, e). More importantly, lncRNA RAET1K silencing also significantly decreased glucose uptake and lactate concentration in HCC cells (Fig. 4f, g). In contrast, RAET1K overexpression increased glucose uptake and lactate concentration in HCC cells (Fig. S5C, D). These data indicate that lncRNA RAET1K serves as an oncogene in HCC cells, possibly through a glycolysis-related mechanism.

\section{The IncRNA RAET1K/miR-100-5p axis modulates glycolysis under hypoxia}

We have revealed that HIF1A overexpression could affect miR-100-5p expression and glycolysis in HCC cells, while HIF1A, IncRNA RAET1K, and miR-100-5p could form a regulatory axis. Next, we evaluated the roles of the 


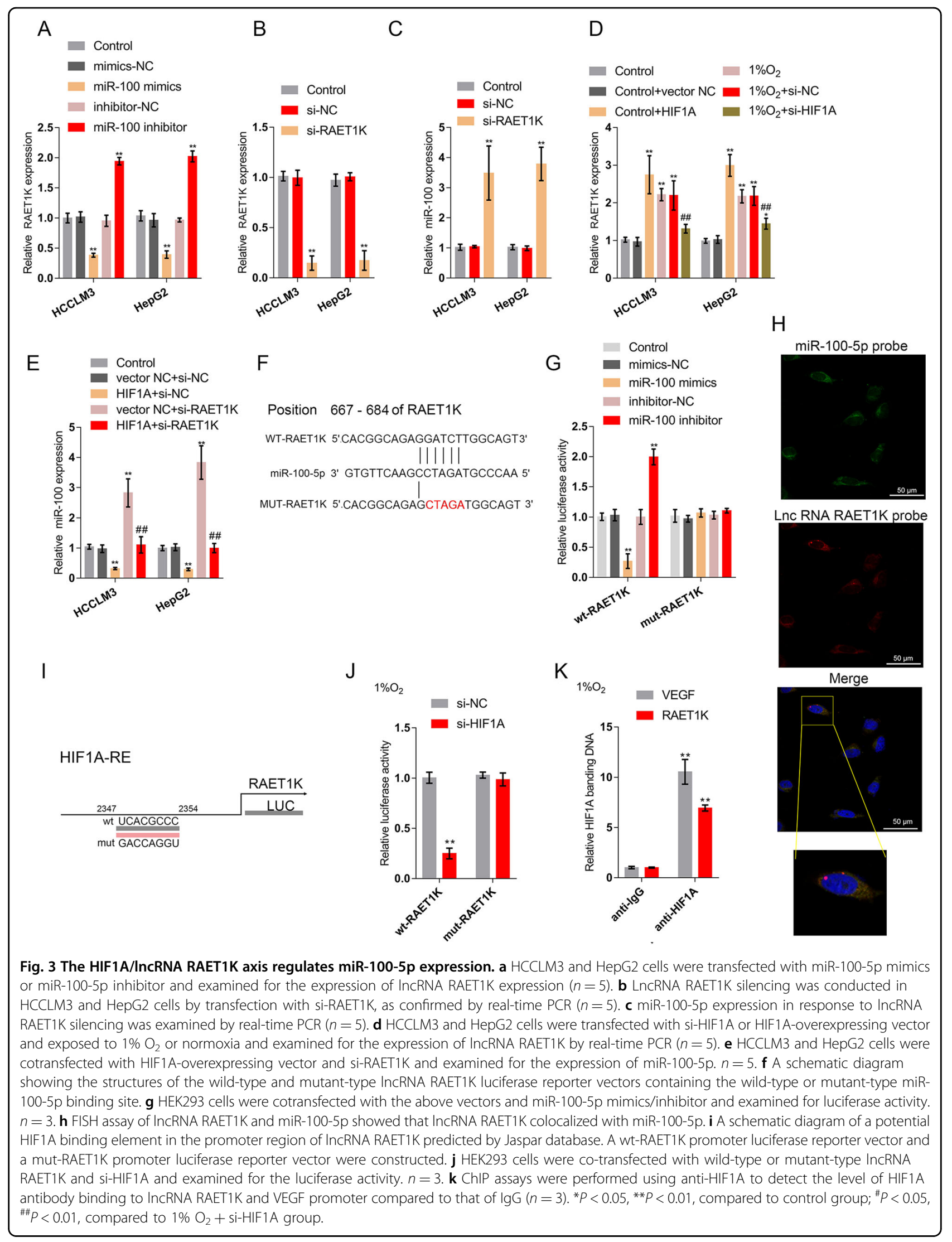




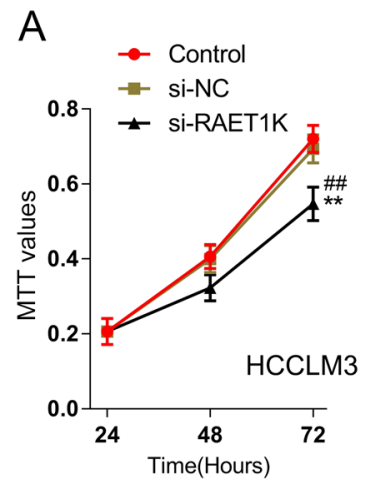

D

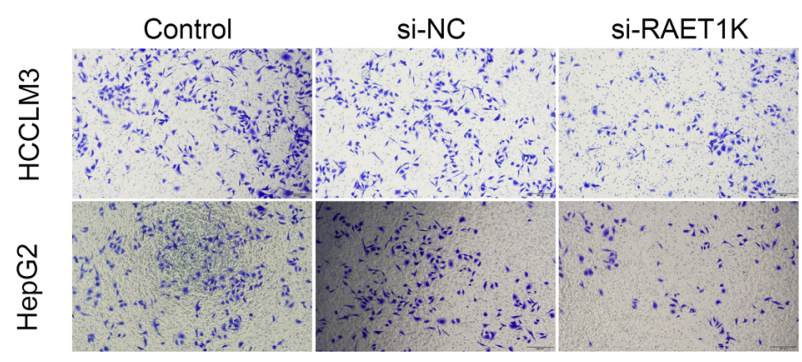

C

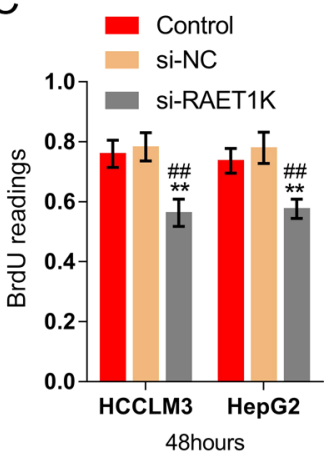

$\mathrm{E}$

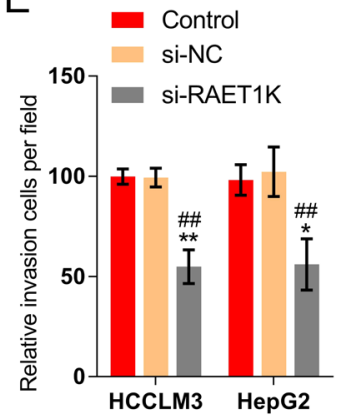

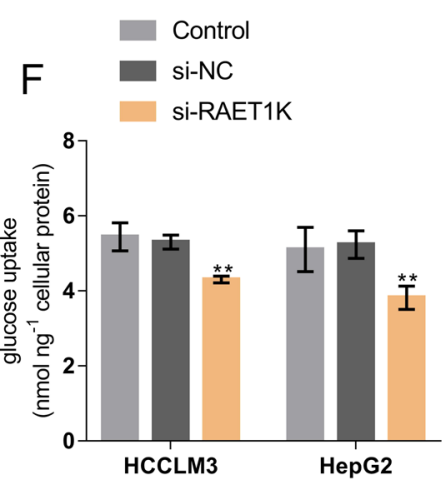

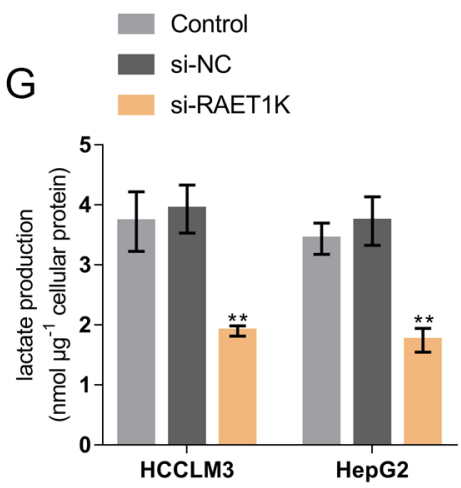

Fig. 4 Effects of IncRNA RAET1K on HCC cell proliferation and invasion. HCCLM3 and HepG2 cells were transfected with si-RAET1K under normoxic conditions and examined for $\mathbf{a}, \mathbf{b}$ cell viability by MTT assays $(n=5), \mathbf{c}$ DNA synthesis capacity by BrdU assays $(n=3)$, $\mathbf{d}$, e cell invasion by Transwell assays, and $\mathbf{f}, \mathbf{g}$ the glucose uptake and lactate concentration $(n=3)$. ${ }^{*} P<0.05,{ }^{* *} P<0.01$, compared to control group.

lncRNA RAET1K/miR-100-5p axis in glycolysis in HCC cells under $1 \% \mathrm{O}_{2}$-induced hypoxia. We cotransfected HCCLM3 and HepG2 cells with si-RAET1K and miR100-5p inhibitor under $1 \% \mathrm{O}_{2}$ exposure and examined lactate concentration, glucose uptake, ATP levels, and LDHA protein were then examined. As previously demonstrated, $1 \% \quad \mathrm{O}_{2}$-induced hypoxia significantly increased, while lncRNA RAET1K silencing decreased lactate concentration, glucose uptake, and the protein levels of LDHA and increased the phosphorylation of AMPK, while under $1 \% \mathrm{O}_{2}$-induced hypoxia, miR-100-5p inhibition significantly reversed the effects of lncRNA
RAET1K silencing on glycolysis and AMPK phosphorylation in HCC cells (Fig. 5a, b, d, e). Accordingly, IncRNA RAET1K silencing significantly reduced, while miR-100$5 p$ inhibition increased the ATP production; the effects of IncRNA RAET1K silencing were reversed considerably by miR-100-5p inhibition (Fig. 5c). In summary, the lncRNA RAET1K/miR-100-5p axis could modulate glycolysis in $\mathrm{HCC}$ cells under $1 \% \mathrm{O}_{2}$-induced hypoxic conditions. To further validate the effect of LncRNA RAET1K/miR-100$5 p$ axis in vivo, we performed HCCLM3 cell xenograft nude mice model. As Fig. 5f-h shown, lncRNA RAET1K knockdown reduced the tumor growth, but miR-100-5p 


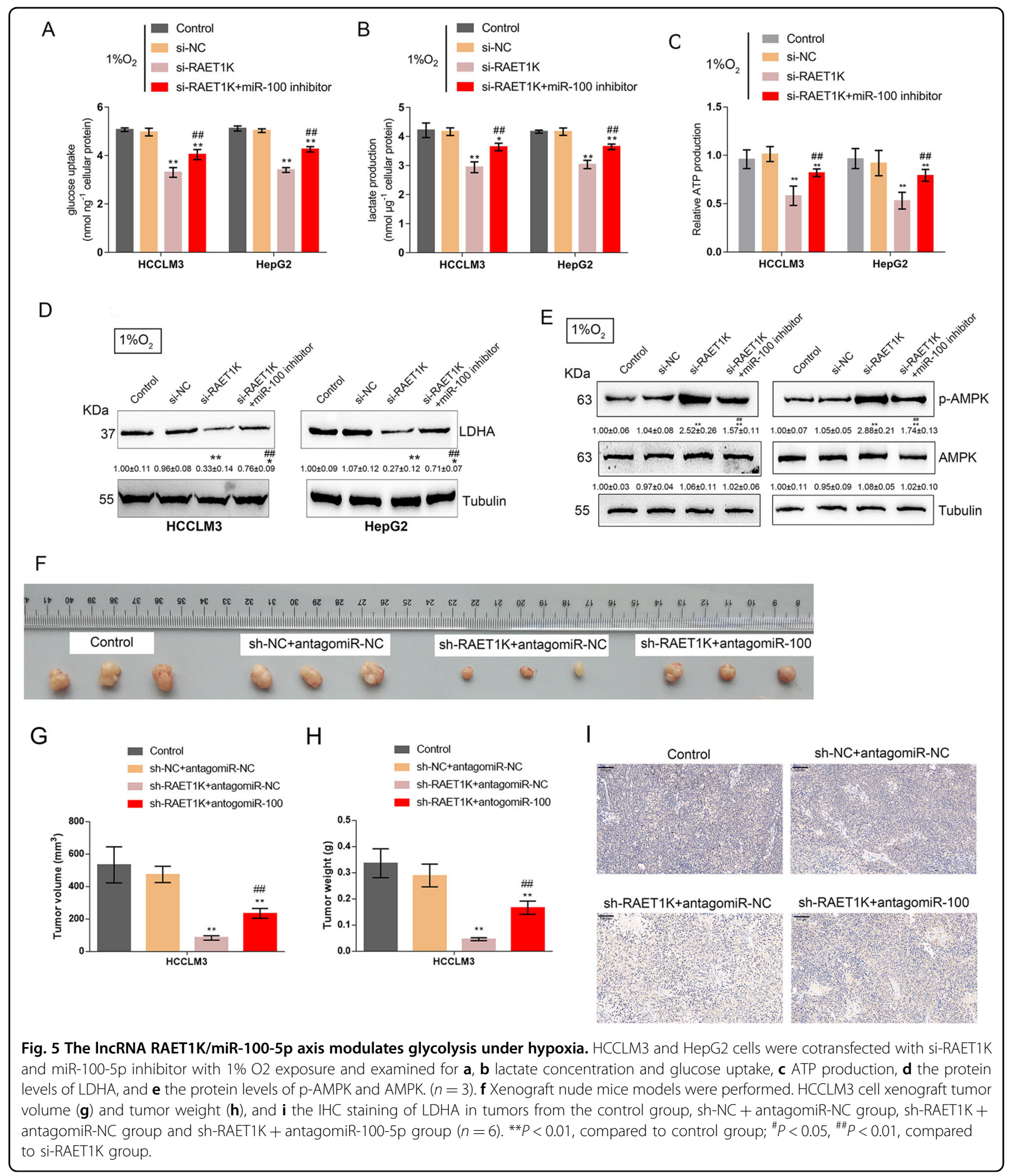

inhibition by antagomiR-100-5p could partly reverse the reduction (Fig. $5 \mathrm{f}-\mathrm{h}$ ). The $\mathrm{IHC}$ staining showed that lncRNA RAETK1K knockdown reduced the LDHA protein expression but miR-100-5p inhibition increased LDHA expression (Fig. 5i).

\section{Expression and correlation of IncRNA RAET1K, LDHA,} HIF1A, and miR-100-5p in tissue samples

As further proof, lncRNA RAET1K, LDHA, and HIF1A expression in tissue samples was examined. According to Fig. 6a-c, the expression of IncRNA RAET1K, LDHA, and 
HIF1A was significantly upregulated in HCC cells compared with nontumor cells. Furthermore, the expression of miR-100-5p within tissue specimens was negatively related to lncRNA RAET1K, LDHA, and HIF1A (Fig. 6d-f), and lncRNA RAET1K, LDHA, and HIF1A were positively correlated with each other (Fig. $6 \mathrm{~g}-\mathrm{i}$ ). The IHC staining results also confirmed that the protein levels of HIF1A and LDHA were higher in tumor tissues, than in normal tissues (Fig. 6j).

\section{Discussion}

Herein, as shown in Fig. 7, we revealed that the expression of miR-100-5p was significantly suppressed in HCC tissue samples and HCC cell lines under hypoxic conditions $\left(\begin{array}{ll}1 \% & \mathrm{O}_{2}\end{array}\right)$. miR-100-5p overexpression significantly suppressed hypoxia-induced increases in lactate concentration and glucose uptake. Hypoxia-induced HIF1A protein expression and reduced miR-100-5p expression while HIF1A silencing significantly rescued miR-100-5p expression upon hypoxia. In addition, hypoxia-induced increases in lactate concentration and glucose uptake were also suppressed by HIF1A silencing. Next, by analyzing the available data in TCGA, we found that lncRNA RAET1K was correlated with HIF1A and miR-100-5p. LncRNA RAET1K could downregulate the expression of miR-100-5p by acting as a sponge, while HIF1A bound the IncRNA RAET1K promoter region to activate its transcription. LncRNA RAET1K silencing significantly suppressed HCC cell proliferation and invasion, and suppressed hypoxia-induced increases in lactate concentration and glucose uptake, while miR-100-5p inhibition reversed the effect of lncRNA RAET1K silencing on hypoxia-induced glycolysis in HCC cells. Finally, the expression of HIF1A, IncRNA RAET1K, and LDHA was upregulated in HCC tissue specimens; the expression of miR-100-5p was negatively related to HIF1A, lncRNA RAET1K, and LDHA; and HIF1A, IncRNA RAET1K, and LDHA were positively correlated with each other.

Recently, it has been revealed that the dysregulation of miR-100-5p contributes to human malignant tumors, such as HCC. Zhou et al. ${ }^{34}$ showed that suppression of miR-100-5p in HCC tissue samples was remarkably related to venous invasion, advanced TNM stage, an incomplete capsule of tumor nodules, reduced cell differentiation, and shorter survival time without recurrence. Moreover, miR-100-5p significantly inhibited HCC cell migration and invasion in vitro, and xenografts of HCC cells with stable expression of miR-100-5p significantly reduced the lung metastasis in vivo. Regarding the molecular mechanism, miR-100-5p suppression upregulates the ICMT-Rac1 signaling pathway, thus promoting HCC cell metastasis. Another group revealed that miR100-5p could promote Atg7-dependent autophagy and subsequent apoptotic cell death. Consistent with these findings, miR-100-5p suppressed HCC cell viability in vivo in mouse xenograft model ${ }^{35}$. During last few decades, aerobic glycolysis has been regarded as a cancer hallmark $^{36}$. Elevated lactate levels are strongly negatively correlated with patient outcomes in many cancers ${ }^{37,38}$. Hence, enhanced glucose consumption may play a critical role in cancer progression. Glycolysis-related genes are ubiquitously overexpressed in cancers ${ }^{39,40}$. Herein, it was demonstrated that under hypoxia, miR-100-5p overexpression dramatically suppressed hypoxia-induced increases in lactate concentration and glucose uptake, indicating that miR-100-5p might affect HCC progression by modulating glycolysis in HCC cells, which adds to previous studies demonstrating that miR-100-5p affects HCC cell proliferation, invasion, and migration. More importantly, HIF1A negatively regulated miR-100-5p expression under hypoxic condition; in addition, HIF1A silencing inhibited hypoxia-induced glycolysis in HCC cells, indicating that deregulation of miR-100-5p could be related to HIF1A function in hypoxia-induced glycolysis in HCC cells.

As we have mentioned, HIF1A is increased in various human malignancies ${ }^{8,9}$. Overexpressed HIF1A could be intimately related to unfavorable prognosis in $\mathrm{HCC}^{41}$. HIF1A dimerizes with HIF1 $\beta$ and activates the transcription of target genes, which exert the significant effects on cancer cell metabolic reprogramming ${ }^{42,43}$. More importantly, HIF1 regulates the transcription of several critical factors in glycolysis ${ }^{11-13}$. So far, the most widely accepted mechanism by which miRNAs exert their biological functions is through interaction with $\operatorname{lncRNAs}{ }^{44,45}$, another type of noncoding RNA. A wide range of previous studies have also indicated that IncRNAs play a critical role in HCC progression ${ }^{46-48}$; however, few studies have investigated the role of lncRNA-mediated glucose metabolism reprogramming in HCC progression ${ }^{49}$. Thus, we attempted to identify lncRNAs that might be related to both HIF1A and miR100-5p and found that IncRNA RAET1K was positively associated with HIF1A but negatively associated with miR-100-5p. As predicted by online tools, HIF1A directly targets the IncRNA RAET1K promoter to activate its transcription while lncRNA RAET1K directly targets miR-100-5p to suppress its expression. These findings indicate that HIF1A, lncRNA RAET1K, and miR-100-5p could form a regulatory axis to modulate glycolysis in HCC to affect HCC progression.

According to the TCGA database, IncRNA RAET1K expression was upregulated in lung adenocarcinoma; Sui et al. ${ }^{50}$ reported a similar result that lncRNA RAET1K was upregulated in lung adenocarcinoma tumor tissues and that lncRNA RAET1K exhibited a significant prognostic value for lung adenocarcinoma. However, the specific role of lncRNA RAET1K in HCC progression has not yet been 


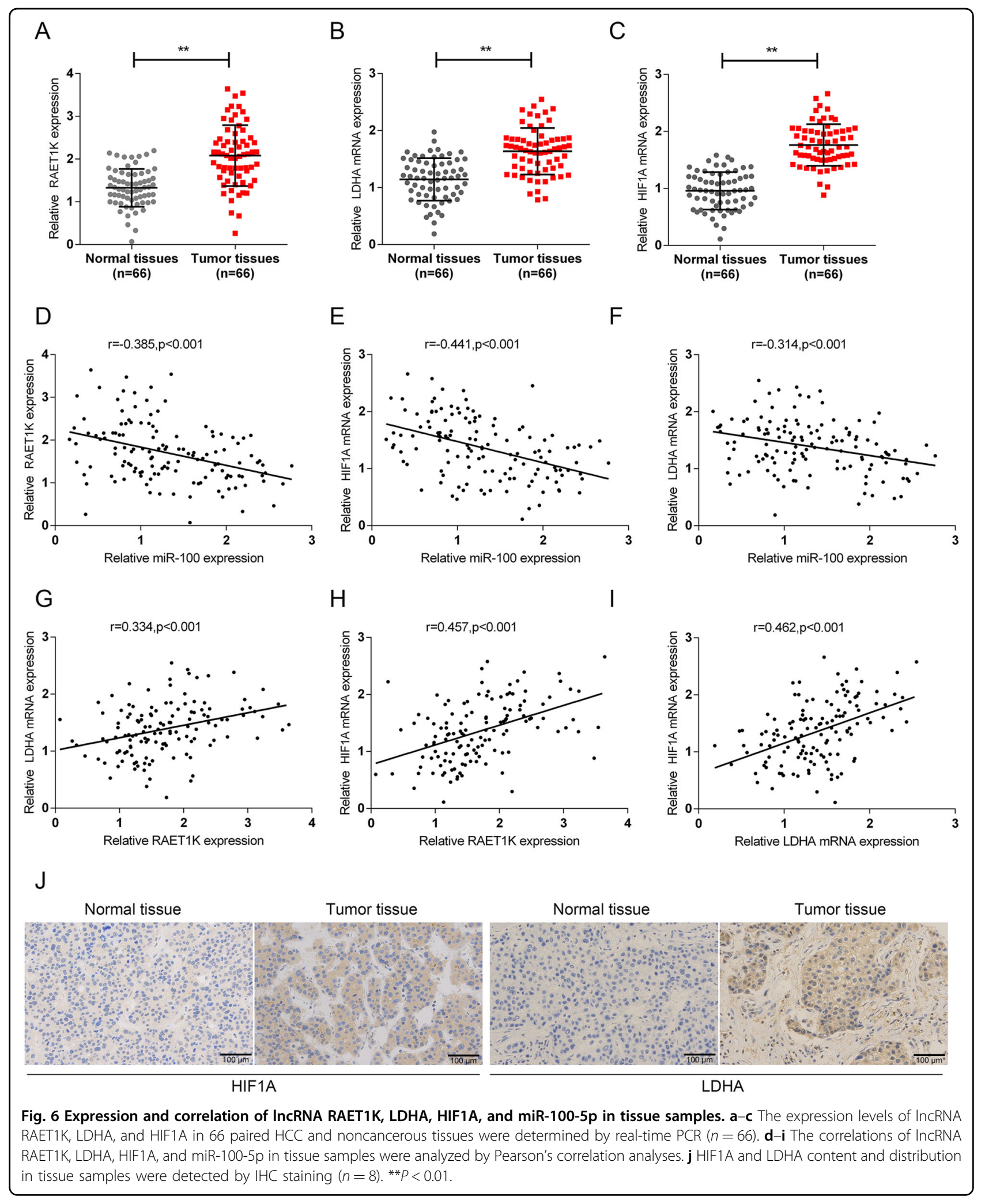

investigated. Herein, we found that lncRNA RAET1K silencing dramatically suppressed the ability of HCC cells to proliferate and invade. Regarding the molecular mechanism, lncRNA RAET1K silencing significantly inhibited while miR-100-5p inhibition enhanced hypoxiainduced increases in lactate concentration and glucose 


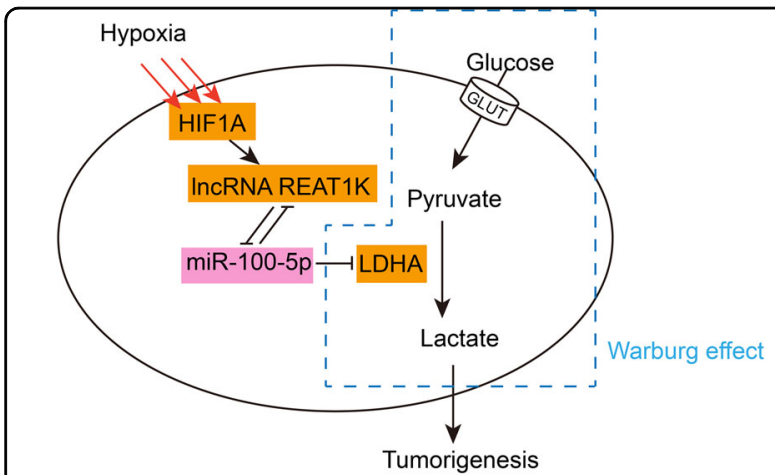

Fig. 7 A schematic diagram of the mechanism. The HIF1A/IncRNA RAET1K/miR-100-5p axis modulates hypoxia-induced glycolysis in HCC cells and then might affect HCC progression.

uptake; more importantly, miR-100-5p suppression significantly attenuated the effects of IncRNA RAET1K silencing on hypoxia-induced glycolysis in HCC cells, indicating that the lncRNA RAET1K/miR-100-5p axis might affect $\mathrm{HCC}$ cell phenotypes by modulating hypoxiainduced glycolysis in HCC cells. As a further confirmation, the expression of HIF1A, IncRNA RAET1K, and LDHA was significantly upregulated in HCC tissues. miR100-5p expression was negatively related to HIF1A, lncRNA RAET1K, and LDHA whereas HIF1A, IncRNA RAET1K, and LDHA could be positively associated with each other. These findings suggest that the lncRNA RAET1K/miR-100-5p axis might also affect HCC progression, which needs further in vivo investigation.

\section{Conclusion}

The HIF1A/lncRNA RAET1K/miR-100-5p axis modulates hypoxia-induced glycolysis in HCC cells and then might affect $\mathrm{HCC}$ progression (Fig. 7).

\section{Conflict of interest}

The authors declare that they have no conflict of interest.

\section{Publisher's note}

Springer Nature remains neutral with regard to jurisdictional claims in published maps and institutional affiliations.

Supplementary Information accompanies this paper at (https://doi.org/ 10.1038/s41419-020-2366-7).

Received: 16 March 2019 Revised: 17 February 2020 Accepted: 18 February 2020

Published online: 09 March 2020

\section{References}

1. Torre, L. A. et al. Global cancer statistics, 2012. CA Cancer J. Clin. 65, 87-108 (2015).

2. Yang, P. et al. TGF-beta-miR-34a-CCL22 signaling-induced Treg cell recruitment promotes venous metastases of HBV-positive hepatocellular carcinoma. Cancer Cell 22, 291-303 (2012).
3. Pavlova, N. N. \& Thompson, C. B. The emerging hallmarks of cancer metabolism. Cell Metab. 23, 27-47 (2016).

4. Warburg, O. On the origin of cancer cells. Science 123, 309-314 (1956).

5. Vander Heiden, M. G., Cantley, L. C. \& Thompson, C. B. Understanding the Warburg effect: the metabolic requirements of cell proliferation. Science $\mathbf{3 2 4}$, 1029-1033 (2009).

6. Hay, N. Reprogramming glucose metabolism in cancer: can it be exploited for cancer therapy? Nat. Rev. Cancer 16, 635-649 (2016).

7. Airley, R. E. \& Mobasheri, A. Hypoxic regulation of glucose transport, anaerobic metabolism and angiogenesis in cancer: novel pathways and targets for anticancer therapeutics. Chemotherapy 53, 233-256 (2007).

8. Palazon, A. et al. An HIF-1alpha/NEGF-A axis in cytotoxic T cells regulates tumor progression. Cancer Cell 32, 669-683 e665 (2017).

9. Bruno, T. et al. Che-1 sustains hypoxic response of colorectal cancer cells by affecting Hif-1alpha stabilization. J. Exp. Clin. Cancer Res. 36, 32 (2017).

10. Porporato, P. E., Dhup, S., Dadhich, R. K., Copetti, T. \& Sonveaux, P. Anticancer targets in the glycolytic metabolism of tumors: a comprehensive review. Front. Pharmacol. 2, 49 (2011).

11. Semenza, G. L. HIF-1: upstream and downstream of cancer metabolism. Curr. Opin. Genet. Dev. 20, 51-56 (2010).

12. Massari, F. et al. Metabolic phenotype of bladder cancer. Cancer Treat. Rev. $\mathbf{4 5}$ 46-57 (2016).

13. Azoitei, N. et al. PKM2 promotes tumor angiogenesis by regulating HIF-1alpha through NF-kappaB activation. Mol. Cancer 15, 3 (2016).

14. Kornienko, A. E., Guenzl, P. M., Barlow, D. P. \& Pauler, F. M. Gene regulation by the act of long non-coding RNA transcription. BMC Biol. 11, 59 (2013).

15. Ge, Y., Zhang, L., Nikolova, M., Reva, B. \& Fuchs, E. Strand-specific in vivo screen of cancer-associated miRNAs unveils a role for miR-21 $\left(^{*}\right)$ in SCC progression. Nat. Cell Biol. 18, 111-121 (2016)

16. Fan, C. et al. Role of long non-coding RNAs in glucose metabolism in cancer. Mol. Cancer 16, 130 (2017).

17. Salmena, L., Poliseno, L., Tay, Y., Kats, L. \& Pandolfi, P. P. A ceRNA hypothesis: the Rosetta Stone of a hidden RNA language? Cell 146, 353-358 (2011).

18. McCarthy, N. Regulatory RNA: layer by layer. Nat. Rev. Genet. 12, 804 (2011).

19. Petrelli, A. et al. Sequential analysis of multistage hepatocarcinogenesis reveals that miR-100 and PLK1 dysregulation is an early event maintained along tumor progression. Oncogene 31, 4517-4526 (2012).

20. Chen, P., Zhao, X. \& Ma, L. Downregulation of microRNA-100 correlates with tumor progression and poor prognosis in hepatocellular carcinoma. Mol. Cell. Biochem. 383, 49-58 (2013).

21. Chen S. C., Chen F. W., Hsu Y. L., Kuo P. L. Systematic Analysis of transcriptomic profile of renal cell carcinoma under long-term hypoxia using next-generation sequencing and bioinformatics. Int. J. Mol. Sci. 18, 2657 (2017).

22. Blick, C. et al. Hypoxia regulates FGFR3 expression via HIF-1alpha and miR-100 and contributes to cell survival in non-muscle invasive bladder cancer. Br. J. Cancer 109, 50-59 (2013).

23. Zhou, S. L. et al. miR-28-5p-lL-34-macrophage feedback loop modulates hepatocellular carcinoma metastasis. Hepatology 63, 1560-1575 (2016).

24. Jin, X. et al. CASC2/miR-24/miR-221 modulates the TRAlL resistance of hepatocellular carcinoma cell through caspase-8/caspase-3. Cell Death Dis. 9, 318 (2018)

25. Ma, J. et al. BMP4 promotes oxaliplatin resistance by an induction of epithelialmesenchymal transition via MEK1/ERK/ELK1 signaling in hepatocellular carcinoma. Cancer Lett. 411, 117-129 (2017).

26. Feng, C. et al. SOX9/miR-130a/CTR1 axis modulates DDP-resistance of cervical cancer cell. Cell Cycle 17, 448-458 (2018).

27. Liu, H., Deng, H., Zhao, Y., Li, C. \& Liang, Y. LncRNA XIST/miR-34a axis modulates the cell proliferation and tumor growth of thyroid cancer through METPI3K-AKT signaling. J. Exp. Clin. Cancer Res. 37, 279 (2018).

28. Valvona, C. J., Fillmore, H. L., Nunn, P. B. \& Pilkington, G. J. The regulation and function of lactate dehydrogenase a: therapeutic potential in brain tumor. Brain Pathol. 26, 3-17 (2016)

29. Wei, S. et al. Promotion of glycolysis by HOTAIR through GLUT1 upregulation via mTOR signaling. Oncol. Rep. 38, 1902-1908 (2017).

30. Li, X. et al. IncRNA Ftx promotes aerobic glycolysis and tumor progression through the PPARgamma pathway in hepatocellular carcinoma. Int. J. Oncol. 53, 551-566 (2018). 
31. Lin, Y. H. et al. Taurine up-regulated gene 1 functions as a master regulator to coordinate glycolysis and metastasis in hepatocellular carcinoma. Hepatology 67, 188-203 (2018).

32. Paraskevopoulou, M. D. \& Hatzigeorgiou, A. G. Analyzing MiRNA-LncRNA Interactions. Methods Mol. Biol. 1402, 271-286 (2016).

33. Ishikawa, A. et al. Edaravone inhibits the expression of vascular endothelia growth factor in human astrocytes exposed to hypoxia. Neurosci. Res. 59, 406-412 (2007).

34. Zhou, H. C. et al. Downregulation of microRNA-100 enhances the ICMT-Racl signaling and promotes metastasis of hepatocellular carcinoma cells. Oncotarget 5, 12177-12188 (2014).

35. Ge, Y. Y. et al. MicroRNA-100 promotes the autophagy of hepatocellular carcinoma cells by inhibiting the expression of mTOR and IGF-1R. Oncotarget $\mathbf{5}$, 6218-6228 (2014).

36. Hanahan, D. \& Weinberg, R. A. The hallmarks of cancer. Cell 100, 57-70 (2000).

37. Brizel, D. M. et al. Elevated tumor lactate concentrations predict for an increased risk of metastases in head-and-neck cancer. Int J. Radiat. Oncol. Biol. Phys. 51, 349-353 (2001).

38. Walenta, S. et al. High lactate levels predict likelihood of metastases, tumor recurrence, and restricted patient survival in human cervical cancers. Cancer Res. 60, 916-921 (2000).

39. Mikuriya, K. et al. Expression of glycolytic enzymes is increased in pancreatic cancerous tissues as evidenced by proteomic profiling by two-dimensional electrophoresis and liquid chromatography-mass spectrometry/mass spectrometry. Int. J. Oncol. 30, 849-855 (2007).

40. Altenberg, B. \& Greulich, K. O. Genes of glycolysis are ubiquitously overexpressed in 24 cancer classes. Genomics 84, 1014-1020 (2004).
41. Liu, L. et al. The impact of high co-expression of Sp1 and HIF1alpha on prognosis of patients with hepatocellular cancer. Oncol. Lett. 12, 504-512 (2016).

42. Rankin, E. B. \& Giaccia, A. J. Hypoxic control of metastasis. Science 352, 175-180 (2016).

43. Li, H., Rokavec, M., Jiang, L., Horst, D. \& Hermeking, H. Antagonistic effects of p53 and HIF1A on microRNA-34a regulation of PPP1R11 and STAT3 and hypoxia-induced epithelial to mesenchymal transition in colorectal cancer cells. Gastroenterology 153, 505-520 (2017).

44. Wan, $X$. et al. The functional sites of miRNAs and IncRNAs in gastric carcinogenesis. Tumour Biol. 36, 521-532 (2015).

45. Yin Q., Feng W., Shen X., Ju S. Regulatory effects of IncRNAs and miRNAs on autophagy in malignant tumorigenesis. Biosci. Rep. 38, 20180516 (2018).

46. Li, C. et al. Progress and prospects of long noncoding RNAs (InCRNAs) in hepatocellular carcinoma. Cell Physiol. Biochem. 36, 423-434 (2015).

47. Mai, H. et al. Molecular pattern of IncRNAs in hepatocellular carcinoma. J. Exp. Clin. Cancer Res. 38, 198 (2019).

48. Zou, H. et al. The role of IncRNAs in hepatocellular carcinoma: opportunities as novel targets for pharmacological intervention. Expert Rev. Gastroenterol. Hepatol. 10, 331-340 (2016).

49. Zheng, Y. L. et al. LINC01554-mediated glucose metabolism reprogramming suppresses tumorigenicity in hepatocellular carcinoma via downregulating PKM2 expression and inhibiting Akt/mTOR signaling pathway. Theranostics $\mathbf{9}$, 796-810 (2019).

50. Sui, J. et al. Molecular characterization of lung adenocarcinoma: a potential four-long noncoding RNA prognostic signature. J. Cell Biochem. 120, 705-714 (2019). 